\title{
QUALIFICANDO - AMBIENTE VIRTUAL DE APRENDIZAGEM PARA ENSINO DE ENGENHARIA DE PRODUÇÃO À DISTÂNCIA VIA INTERNET
}

\section{QUALIFICANDO - A WEB-BASED VIRTUAL ENVIRONMENT FOR INDUSTRIAL ENGINEERING DISTANCE LEARNING}

\author{
Marcelo Nogueira Cortimiglia - cortimiglia@producao.ufrgs.br \\ Flávio Sanson Fogliatto - ffogliatto@producao.ufrgs.br \\ Leandro Vettorazzi Gabrieli - gabriell@producao.ufrgs.br \\ Icaro Paulo Ludwig - icaro@producao.ufrgs.br \\ Departamento de Engenharia de Produção e Transportes - DEPROT \\ Programa de Pós-Graduação em Engenharia de Produção - PPGEP \\ Universidade Federal do Rio Grande do Sul - UFRGS \\ CEP 90040-020 - Porto Alegre, RS, Brasil
}

\begin{abstract}
Resumo
Este artigo relata a experiência de desenvolvimento de um Ambiente Virtual de Aprendizagem baseado na Internet para o ensino à distância de Engenharia de Produção, designado por Qualificando. A estrutura do ambiente, elaborado a partir de tecnologias de banco de dados e programação dinâmica de páginas de Internet, é descrita no contexto da retrospectiva histórica do projeto. Nesse sentido, são discutidos aspectos técnicos e gerenciais relacionados com as soluções adotadas durante o processo de otimização e melhoria contínua do ambiente, apresentados resultados das modificações efetuadas e propostas futuras linhas de desenvolvimento e pesquisa associadas com a iniciativa.
\end{abstract}

Palavras-chave: Educação à Distância, Ambiente Virtual de Aprendizagem, Internet.

\begin{abstract}
This work describes the development experience of an Internet-based Virtual Learning Environment for Industrial Engineering distance teaching named Qualificando. The environment's structure, which was developed through database technology and dynamic web-programming language, is described in relation to the project's historical perspective. In this context, technical and management issues related to the solutions adopted throughout the environment's optimization and continual improvement processes are discussed, and the results of the modifications performed during these processes are presented along with future development and research suggestions.
\end{abstract}

Key-words: Distance Education, Virtual Learning Environment, Internet.

\section{Introdução}

As novas tecnologias de informação e comunicação têm revolucionado diversos aspectos da vida moderna. Por um lado, ajudam a dar forma para uma sociedade verdadeiramente 
globalizada, na qual a velocidade de obsolescência dos conhecimentos aplicados e das informações é significativa. Por outro lado, possibilitaram o surgimento e facilitam a operacionalização de um paradigma educacional focado no estudante e baseado em versatilidade e flexibilidade de meios e técnicas. Tal paradigma, por sua vez, permite ao aprendente conciliar seu estudo com atividades profissionais e familiares cotidianas, o que Tight (1998), Lévy (1999), Candy (2000) e Bork (2001) denominam “aprendizado ao longo da vida". O ensino-aprendizagem à distância, com suas abordagens e soluções tecnológicas, constitui-se em uma das principais vertentes deste paradigma de aprendizado continuado.

A Internet é um dos expoentes da revolução das tecnologias de informação e comunicação. Para Keen (1996), a Internet se constitui no "núcleo de toda uma cultura, e quase um culto". Em função de seu alcance e popularidade (BRADSHAW, 2001; NGANI et al., 2002; AIKEN et al., 2003), é compreensível seu emprego como mídia para o ensino-aprendizagem à distância, constituindo-se no que Garrison (1985), Moore e Kearsley (1996) e Mena et al. (2001) consideram a base tecnológica da terceira geração de ensino à distância. Hoje, a importância e aceitação da Internet como tecnologia educacional já se encontram estabelecidas, conforme atestam pesquisas realizadas junto a instituições de ensino e organizações de negócios (LEWIS et al., 1999; U.S DEPARTMENT OF EDUCATION, 1999, 2002; VODANOVICH e PIOTROWSKI, 2001; EISENBARTH, 2003). Além disso, argumenta-se que o uso da Internet como mídia para o ensino-aprendizagem à distância é fundamental na democratização do acesso ao conhecimento nos países em desenvolvimento (DRUCKER, 2000; MUTULA, 2002; ARMENGOL, 2002).

Segundo relatório da Web-Based Education Commission (2000), o recente crescimento do ensino à distância via Internet foi possível graças ao aumento da capacidade de transmissão de dados nas linhas de telecomunicação, expansão dos serviços de conexão à Internet via banda larga e diminuição dos custos de conexão. Nos próximos anos, tais tendências facilitadoras da difusão da Internet como mídia educativa devem continuar se manifestando (AYRES e WILLIAMS, 2004), resultando em uma provável manutenção ou expansão da participação desta mídia no ensino à distância.

Entre os recursos de Internet empregados em ensino à distância, destacam-se o correio eletrônico, grupos e fóruns de discussão, salas de bate-papo, ferramentas de conferência virtual, teleconferência, videoconferência, dataconferência, serviços de mensagens instantâneas e aplicativos de trabalho colaborativo (groupware). Além destes recursos, existem aplicativos de Internet especificamente desenvolvidos para uso educacional, entre os 
quais se destacam os Ambientes Virtuais de Aprendizagem (AVAs). O desenvolvimento de AVAs para o ensino à distância tem sido fértil campo de pesquisa acadêmica nos últimos anos; ver, por exemplo, Slator et al. (1999), Hiddink (2001), Bouras et al. (2001), Schlemmer e Fagundes (2001), Sheremetov e Arenas (2002), Britain e Liber (2004) e Sivakumar e Robertson (2004).

Este artigo relata o processo de desenvolvimento de um AVA, denominado "Qualificando", apropriado para o ensino-aprendizagem à distância via Internet de Engenharia de Produção. Mais especificamente, o artigo reporta a evolução de um AVA originalmente destinado ao ensino de uma ferramenta de informática para englobar disciplinas características de um curso de Engenharia de Produção. A relevância do trabalho pode ser justificada (i) pela crescente utilização de AVAs em diferentes áreas do conhecimento, conforme reportado acima e (ii) pela descrição do processo de elaboração de um AVA que utiliza como base uma experiência de sucesso, também detalhada no trabalho. Espera-se, assim, que o trabalho contribua para o desenvolvimento de ambientes similares de aprendizagem virtual.

\section{Fundamentação teórica}

Segundo Schlemmer e Fagundes (2001), os AVAs são sistemas para gerenciamento de aprendizagem via $W e b$, os quais "sintetizam a funcionalidade de software para comunicação mediada por computador (CMC) e métodos de entrega de material de cursos online". O diferencial dos AVAs em relação a material para auto-estudo disponibilizado na $\mathrm{Web}$, como livros e apostilas eletrônicas, é o foco nas interações estudante-estudante e estudante-instrutor.

Entre os AVAs predominam, conforme Britain e Liber (2004), sistemas baseados em arquitetura cliente-servidor: os computadores servidores disponibilizam ambientes informatizados acessados via $\mathrm{Web}$ por navegadores nos computadores clientes. Dentre as funcionalidades mais comuns nos AVAs, listam-se: mural ou quadro de notícias, lista de participantes, acesso às páginas $W e b$ pessoais dos participantes, ferramentas de colaboração síncrona, cronograma e conteúdo instrucional do curso, ferramentas de busca, lista de endereços eletrônicos da equipe de tutoria e dos alunos, ferramentas de conferência virtual, avaliações e testes, marcadores e editores de conteúdo, módulo de navegação, repositório de recursos em multimídia e área de disponibilização de arquivos.

Os usuários de AVAs são usualmente classificados como Estudantes e Tutores. Ambos costumam dispor de acesso às ferramentas de comunicação e interação, mas os Tutores geralmente dispõem de recursos privilegiados de autoria de material instrucional e 
acompanhamento das atividades. Estudantes, por sua vez, concentram suas atividades nos módulos de navegação e, por vezes, dispõem de recursos de comunicação privada de acesso restrito (BRITAIN e LIBER, 2004). É importante observar que, dada a importância do diálogo e da interação social para um efetivo aprendizado, a maioria dos AVAs é estruturada de modo a facilitar todo o tipo de comunicação e colaboração ativa entre os agentes do processo pedagógico (CLARK, 2001), freqüentemente fazendo uso de aplicativos de groupware e ferramentas de comunicação mediada por computador (TU, 2000; CHOU, 2003).

Diversas pesquisas têm sido conduzidas a respeito do desenvolvimento de AVAs. Nachmias et al. (1999) desenvolveram uma taxonomia para classificação destas iniciativas educacionais na Internet a partir de um estudo de cerca de 500 projetos de ensino à distância na Internet, possibilitando o mapeamento das tendências de evolução dos AVAs. Esta iniciativa é importante pois, em conseqüência do volume de pesquisa e interesse na área, diferentes modelos de AVAs têm sido propostos. Hiddink (2001), por exemplo, apregoa uma arquitetura de base de dados e tecnologia XML para separação da estrutura de conteúdo e layout de apresentação. Dessa forma, torna-se possível o uso do mesmo sistema em diferentes configurações de apresentação conforme necessidades dos usuários e restrições de tecnologia, maximizando a usabilidade e oportunidades de reutilização do material instrucional.

Do mesmo modo, é possível desenvolver AVAs especialmente voltados para o ensino de uma área do conhecimento ou disciplina específica. Como exemplo, tem-se o trabalho de Slator et al. (1999), que apresenta sistemas para ensino à distância via Internet de disciplinas tão distintas como Geociências, Ciências da Computação, História, Economia e Antropologia.

A proposta do AVA Qualificando combina estas duas abordagens, uma vez que trata-se de uma iniciativa para ensino de disciplinas relacionadas com a Engenharia de Produção, particularmente em caráter de ensino de extensão voltado para estudantes adultos interessados em capacitação contínua, e que faz uso de uma arquitetura baseada em linguagens dinâmicas de programação para a Internet associadas com tecnologias de bases de dados.

\section{Qualificando: Ambiente Virtual de Aprendizagem de Engenharia de Produção}

Em sua versão atual, o AVA Qualificando é o resultado da evolução de uma proposta iniciada em 2001 com o Curso On-line de Base de Dados na Gestão Empresarial (CORTIMIGLIA e OLIVEIRA, 2002; MÜLLER et al., 2003). Ao longo da história deste curso, oferecido totalmente via Internet em caráter de curso de extensão pelo Programa de Pós-Graduação em 
Engenharia de Produção (PPGEP) da Universidade Federal do Rio Grande do Sul, foram constituídas vinte turmas, totalizando mais de 370 alunos matriculados até o encerramento das atividades no fim de 2003. Para esta iniciativa, foi desenvolvido na época um AVA, via Internet, constituído na percepção do usuário externo, por um subsistema de interface com o usuário e um subsistema de suporte ao aprendizado (OLIVEIRA e CORTIMIGLIA, 2003).

O primeiro subsistema englobava ferramentas de reconhecimento e validação dos dados de usuários, bem como instrumentos de coleta e análise de dados relativos ao andamento das atividades pedagógicas e avaliações de aprendizado. Por meio desse subsistema, também, o material instrucional, devidamente formatado e adaptado à proposta pedagógica do projeto de ensino à distância, era transmitido ao aluno. A partir dos dados coletados nas interfaces do subsistema, gerava-se informação para o ajuste contínuo do conteúdo do curso e para a adequação das práticas pedagógicas às necessidades dos alunos. Esta abordagem participativa ia ao encontro de uma premissa de projeto e desenvolvimento tanto do ambiente pedagógico quanto do conteúdo instrucional focados no usuário, a qual, segundo Blythe (2001), possibilita engajar os estudantes como participantes ativos, conseqüentemente ampliando a usabilidade do sistema e, indiretamente, a eficácia do aprendizado. Nesse sentido, os instrumentos e métodos de avaliação de satisfação empregados foram de significativa valia para o aprimoramento do ambiente de aprendizagem (CORTIMIGLIA e OLIVEIRA, 2002).

O subsistema de suporte ao aprendizado, por sua vez, era formado por instrumentos de comunicação síncrona (sala de bate-papo, sala de palestras e sistemas de mensagens instantâneas) e assíncrona (fórum, mural de recados e atendimento via e-mail), além de mídias alternativas ao texto escrito (vídeos, sons e animações), as quais eram utilizadas na transmissão da informação no subsistema de interface com o usuário. Sua composição básica permitia a máxima interação entre alunos e tutores, possível através da rede mundial de computadores, desde o momento da matrícula até a emissão do certificado de conclusão.

Um terceiro subsistema, de caráter auxiliar e de acesso restrito à equipe de Tutoria, também estava presente no AVA original. Tratava-se de um subsistema de gerenciamento, dividido em um módulo administrativo, destinado à operacionalização remota das atividades de gestão do curso, e um módulo pedagógico, associado ao acompanhamento do processo de ensinoaprendizagem pelos Tutores. Em relação ao acompanhamento pedagógico, era possível monitorar, por exemplo, resultados comparativos das avaliações de aprendizado de um aluno, ou turma de alunos, em relação aos demais. Alternativamente, os aplicativos do subsistema permitiam medir os tempos de acesso a cada trecho do material instrucional e das avaliações. 
Por meio do módulo pedagógico, os Tutores também podiam dispôr de uma série de indicadores de participação elaborados a partir do número de acessos e tempo de conexão dos alunos às ferramentas de interação e comunicação. Entre as ferramentas do módulo administrativo estavam incluídos aplicativos para a geração de relatórios de suporte à gestão como, por exemplo, estatísticas atualizadas sobre a composição das turmas, indicadores de retorno das iniciativas de divulgação e relatórios para acompanhamento e operacionalização da gestão financeira. Finalmente, o módulo administrativo permitia efetuar a manutenção técnica do AVA. Todos os subsistemas eram integrados em uma interface Web construída dinamicamente através de tecnologias de bases de dados e linguagens de programação de páginas na Internet. A figura 1 representa esquematicamente a estrutura do AVA original do Curso On-line de Base de Dados na Gestão Empresarial.

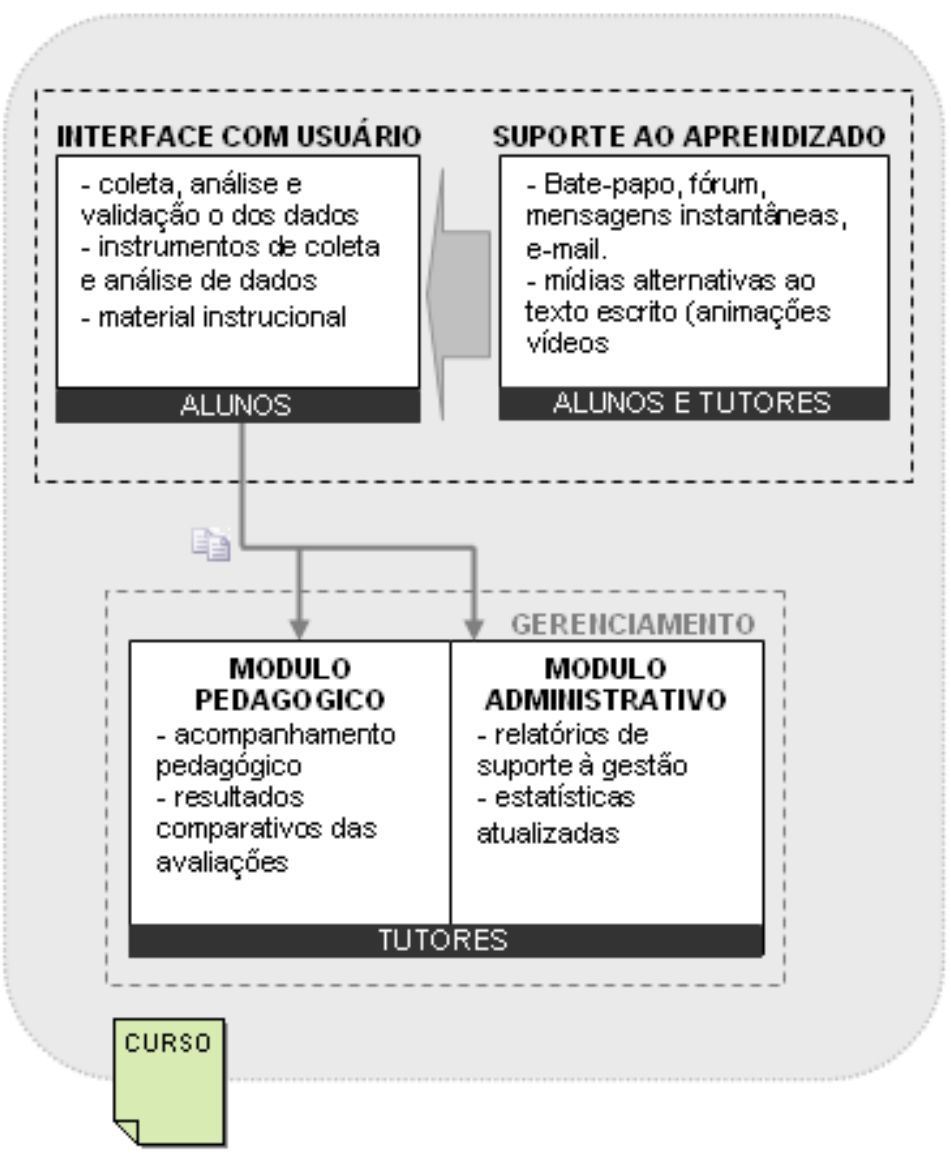

Figura 1 - Estrutura funcional do AVA do Curso On-line de Base de Dados na Gestão Empresarial.

O AVA original, empregado no Curso On-line de Base de Dados na Gestão Empresarial até 2003, foi totalmente elaborado por equipes do PPGEP. A opção pelo desenvolvimento interno se deu, principalmente, a fim de garantir independência em relação aos atuais programas educacionais comerciais, permitindo total domínio da tecnologia para atender a necessidades por eventuais modificações no sistema. Além do sistema de ensino propriamente dito, 
compreendido pelo AVA, também foi desenvolvido internamente todo o conteúdo instrucional do referido curso. Tal conteúdo foi elaborado a partir de extensa pesquisa e adaptado ao formato de hipertexto enriquecido com multimídia apropriado para transmissão a partir do AVA.

A iniciativa do Curso On-line de Base de Dados na Gestão Empresarial foi de vital importância no desenvolvimento de recursos humanos e metodologias de trabalho apropriadas para ofertar ensino à distância via Internet, os quais foram plenamente aproveitados na empreitada do novo AVA, o Qualificando. Neste projeto, o Ambiente Virtual de Aprendizagem originalmente estruturado para o Curso On-line de Base de Dados na Gestão Empresarial foi consolidado e expandido através da incorporação de um módulo de autoria de conteúdos instrucionais e outro de suporte ao gerenciamento de múltiplos cursos.

Todos os subsistemas anteriormente presentes no AVA original foram remodelados de modo a terem seu desempenho e usabilidade aprimorados. Os códigos de programação passaram por um processo de revisão significativo a fim de melhorar o desempenho técnico do AVA, especialmente no subsistema de interface com o usuário. As ferramentas de coleta de dados também foram otimizadas a fim de eliminar falhas técnicas. Por meio do uso de linguagens dinâmicas de programação na $W e b$ mais avançadas, por exemplo, tornou-se possível coletar dados de tempo de acesso ao conteúdo instrucional com uma precisão da ordem de segundos, enquanto no sistema antigo a grandeza utilizada neste mesmo indicador era da ordem de dezenas de minutos. Da mesma forma, a estrutura da base de dados do AVA foi reprojetada a fim de eliminar inconsistência e duplicidade nos dados armazenados.

Outro aspecto do subsistema de interface com o usuário ${ }_{2}$ que foi radicalmente revisado ${ }_{2}$ foi a apresentação estética e funcional das páginas que constituem o AVA. De um total de 114 recomendações presentes no estudo ergonômico de Reitz (2003) sobre o website do Curso de Base de Dados, foi possível identificar dezenas de oportunidades de melhoria no leiaute das páginas, as quais foram implementadas no Qualificando. Códigos visuais, significações textuais, ícones e indicações gráficas foram remodelados e padronizados em todo o AVA, de modo a facilitar a execução de determinadas ações e, desta forma, melhorar a usabilidade geral do sistema. Objetivou-se, também, diminuir o número de ações por parte dos usuários, reduzindo com isso a carga de trabalho cognitivo associada à navegação nas páginas. As Figuras 2 e 3 ilustram a extensão das modificações operadas na apresentação gráfica do AVA através da comparação das páginas de abertura do AVA original, na Figura 2, e do AVA Qualificando, na figura 3. 
ISSN 1676 - 1901 / Vol. 5/ Num. 2/ Junho de 2005

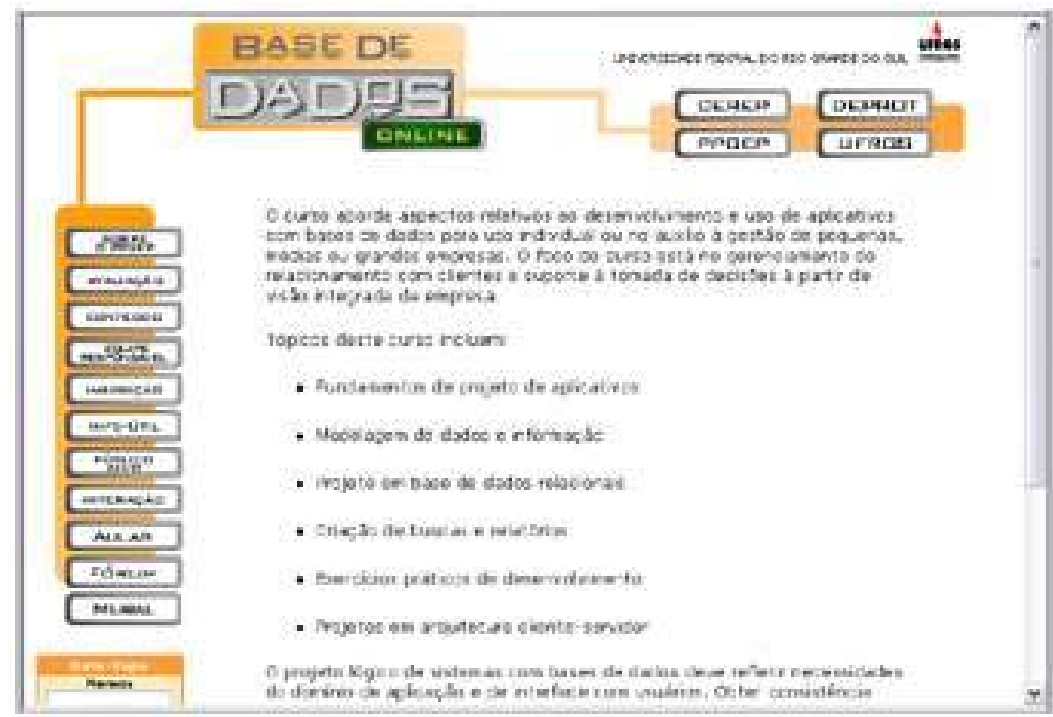

Figura 2 - Evolução da apresentação gráfica da tela incial: AVA original.

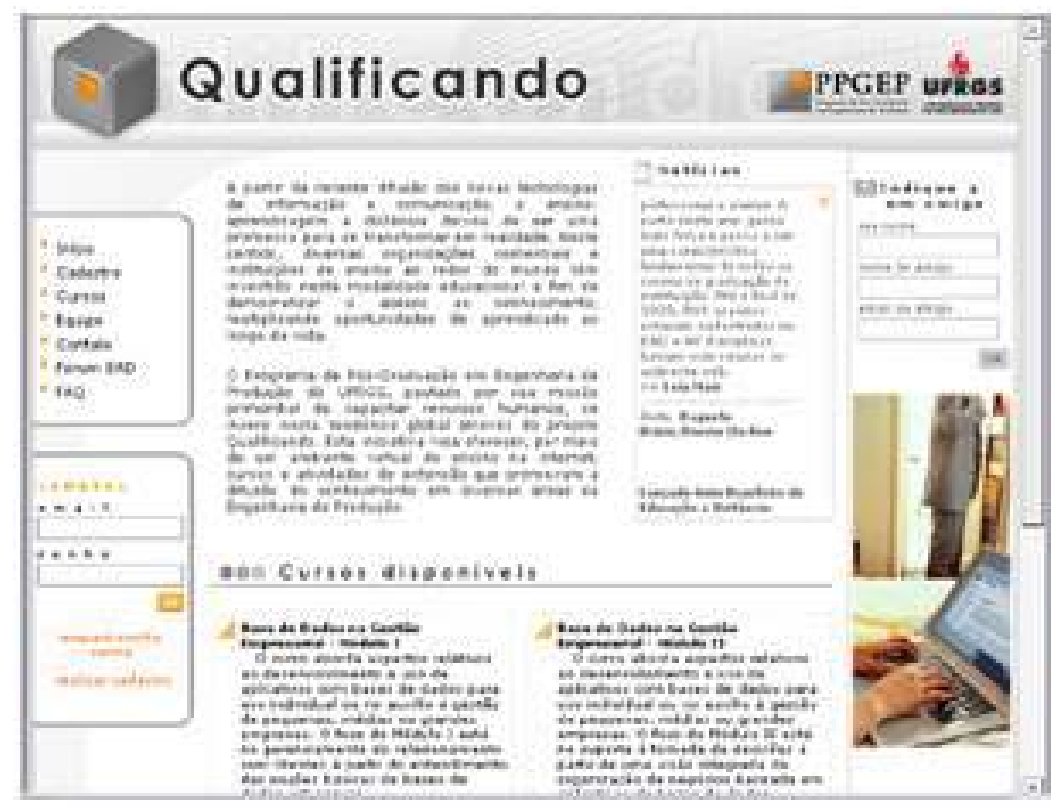

Figura 3 - Evolução da apresentação gráfica da tela incial: AVA Qualificando.

Por meio do módulo de gerenciamento de múltiplos cursos, tornou-se possível que múltiplos usuários administrem e acompanhem as atividades de mais de um curso ou disciplina, aumentando a flexibilidade das iniciativas pedagógicas oferecidas pelo sistema e a capacidade do mesmo. Todas as ferramentas do subsistema de gerencimento do AVA original, tanto do módulo pedagógico quanto do módulo administrativo, foram aprimoradas para utilização em múltiplos cursos. Fez-se necessário, entretanto, adicionar ferramentas para cadastro e controle dos usuários internos do sistema, cada qual responsável por diferentes atividades no AVA como autoria de cursos, tutoria de turmas ou manutenção técnica das páginas.

Por outro lado, foi agregado um módulo de autoria pedagógica, o qual objetiva permitir a 

criação de atividades e conteúdos instrucionais em hipertexto enriquecido com multimídia, de modo que estes sejam compatíveis com os outros subsistemas do AVA. Dentro da mesma filosofia centrada no usuário empregada no AVA original, o módulo de autoria foi projetado para ser utilizado mesmo por usuários pouco familiarizados com as linguagens de programação na $W e b$.

A figura 4 sintetiza a estrutura do AVA Qualificando, realçando a capacidade de gerenciamento de múltiplos cursos e a introdução do módulo de autoria na estrutura funcional do sistema.

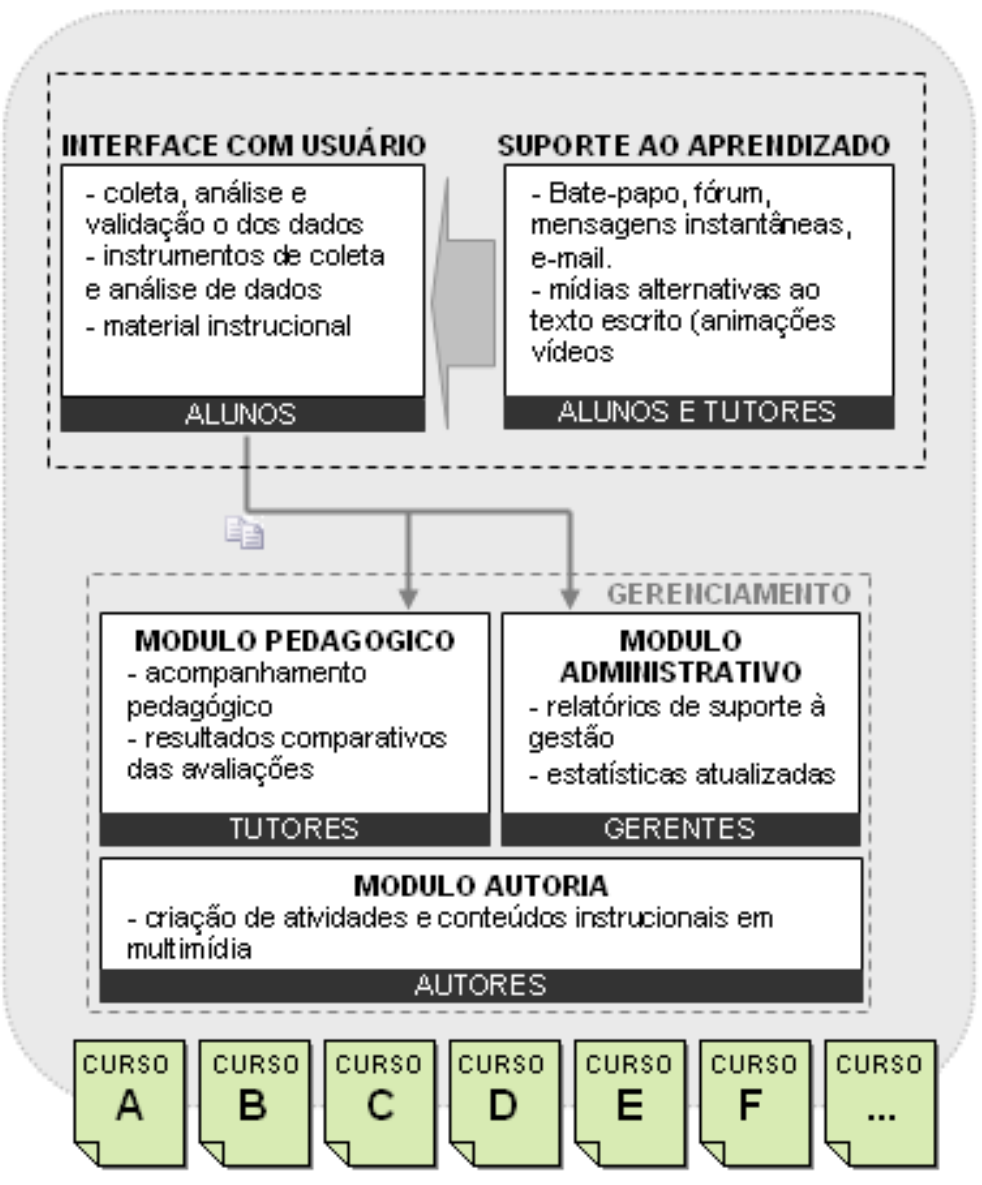

Figura 4 - Estrutura funcional do AVA Qualificando

\section{Resultados}

Atualmente, o AVA Qualificando encontra-se em fase de teste, tendo iniciado suas atividades oficiais em Novembro de 2003 com uma nova versão introdutória do Curso On-line de Base de Dados na Gestão Empresarial. A fim de verificar as funcionalidades do subsistema de gerenciamento de múltiplos cursos, foram desenvolvidos também $_{2}$ conteúdos instrucionais para uma versão avançada do referido curso, além de cursos sobre redes de computadores e aplicativos de edição de texto para publicações acadêmicas. 
Junto aos usuários internos (autores de conteúdo, administradores e tutores dos cursos), os principais pontos negativos da avaliação do novo AVA estão relacionados ao subsistema de gerenciamento. Entre as principais reclamações destes usuários, coletadas através de entrevistas informais semi-estruturadas, pode-se citar a excessiva carga de trabalho associada à criação de um novo curso, tanto em relação ao desenvolvimento do material instrucional per se quanto aos aspectos administrativos como planejamento das atividades, gestão das inscrições e supervisão das turmas. Da mesma forma, os autores não consideram a utilização do módulo de autoria tão intuitiva quanto os projetistas do AVA tencionavam. Tal deficiência do sistema pode estar relacionada tanto à falta de conhecimento dos autores a respeito de linguagens de programação para Internet quanto à complexidade da ferramenta de autoria. Estudam-se medidas corretivas para esta questão, as quais envolvem a remodelagem das interfaces de modo a diminuir a carga de trabalho dos autores e a instituição de um módulo de ajuda on-line destinado a esclarecer os principais conceitos envolvidos no desenvolvimento do material instrucional.

Também têm sido realizadas avaliações do novo AVA junto aos usuários externos, ou seja, os alunos. Até o presente momento, cerca de 40 alunos efetuaram inscrição. Estão sendo colhidas avaliações de usabilidade do sistema, satisfação e aprendizado dos alunos, dados sobre o desempenho técnico e sugestões qualitativas sobre as funcionalidades do AVA. As avaliações de satisfação são compostas por entrevistas semi-estruturadas e questionários quantitativos, cujo instrumento é idêntico ao empregado no AVA original a fim de permitir comparação entre os dois ambientes (CORTIMIGLIA e OLIVEIRA, 2002). As informações coletadas são comparadas com os critérios de otimização utilizados no projeto do novo sistema. Os resultados até o momento têm sido extremamente positivos. De modo geral, os índices de satisfação com a relevância e aplicabilidade do conteúdo, conhecimentos novos adquiridos, planejamento das aulas e qualidade do material didático têm se mantido nos mesmos níveis da versão anterior. Observou-se, entretanto, uma redução significativa nas reclamações referentes a falhas técnicas no acesso e uso do sistema. Da mesma forma, importantes considerações têm sido encaminhadas pelos alunos através de um instrumento aberto para coleta de sugestões que acompanha os questionários de avaliação de satisfação. Por meio deste instrumento, foi possível identificar problemas no procedimento de inscrição nos cursos. De fato, uma vez que o usuário precisa antes efetuar seu cadastro no AVA para, posteriormente, escolher os cursos e turmas de seu interesse, o número de passos e ações necessárias para o cadastramento cresceu significativamente em relação ao sistema original de 
apenas um curso.

\section{Conclusões e Futuros Trabalhos}

Em função da ampla aceitação da Internet como mídia educacional, as perspectivas de desenvolvimento de AVAs inovadores baseados na Web são positivas. Neste artigo, apresentou-se o Qualificando, um AVA para o ensino à distância via Internet de Engenharia de Produção. Trata-se de uma experiência bem sucedida de ensino à distância, comprovada pela sua boa aceitação junto a autores, tutores e alunos.

Como desdobramento futuro do desenvolvimento aqui reportado, espera-se utilizar o AVA Qualificando como base de um sistema virtual de ensino capaz de apoiar a oferta de disciplinas de pós-graduação e graduação do Departamento de Engenharia de Produção da UFRGS. Para tanto, é necessário testar e avaliar de modo mais intenso e crítico os aspectos relacionados com o subsistema de gerenciamento de múltiplos cursos e, conseqüentemente, otimizar as interfaces de acesso e controle de múltiplos usuários. Da mesma forma, o volume de acesso simultâneo ao sistema tende a aumentar proporcionalmente ao número de alunos matriculados, e poucos estudos sobre o desempenho técnico do sistema sob grandes volumes de acesso foram efetuados até o presente momento. É fundamental, também, estender as avaliações de satisfação para outros perfis de público-alvo que caracterizem mais fielmente o tipo de aluno que se espera atender futuramente com o Qualificando.

\section{Referências}

AIKEN, M.; VANJANI, M.; BAISHALI, R. \& MARTIN, J. (2003) - College student Internet use. Campus-Wide Information Systems, Vol. 20, $\mathrm{n}^{\circ}$ 5, pp. 182-185.

ARMENGOL, M. C. (2002) - Global and Critical Visions of Distance Universities and Programs in Latin America. International Review of Research in Open and Distance Learning. Vol. 3, $\mathrm{n}^{\circ}$ 2. Disponível on-line em http://www.irrodl.org/content/v3.2/armengol.html, último acesso em 24/03/2004.

AYRES, R.U.; WILLIAMS, E. (2004) - The digital economy: Where do we stand? Technological Forecasting \& Social Change, Vol. 71, p. 315-339.

BLYTHE, S. (2001) - Designing online courses: User-centered practices. Computers and Composition, Vol. 18, pp. 329-346.

BORK, A. (2001) - Adult education, lifelong learning, and the future. Campus-Wide Information Systems, Vol. $18, \mathrm{n}^{\circ}$, pp. 195-203.

BOURAS, C.; PHILOPOULOS, A.; TSIATSOS, T. (2001) - e-Learning through distributed virtual environments. Journal of Network and Computer Applications, vol. 24, pp. 175-199.

BRADSHAW, A. C. (2001) - Inernet Users Worldwide. Educational Techology Research and Development, 
Vol. $49, \mathrm{n}^{\circ} 4$, pp. 112-117.

BRITAIN, S.; LIBER, O (2004) A Framework for Pedagogical Evaluation of eLearning Environments. Bolton: Bolton Institute, 2004. Disponível on-line em http://www.cetis.ac.uk/members/ pedagogy/files/4thMeet_framework/VLEfullReport, ultimo acesso em 15/04/2005.

CANDY, P.C. (2000) - Reaffirming a proud tradition. Active Learning in Higher Educ., Vol. 1, No. 2, pp. 101-125.

CHOU, C. (2003) - Interactivity and interactive functions in web-based learning systems: a technical framework for designers. British Journal of Educational Technology. Vol. 34, nº 3, pp. 265-279.

CLARK, J. (2001) - Stimulating collaboration and discussion in online learning environments. Internet and Higher Education. Vol. 4, pp. 119-124.

CORTIMIGLIA, M. N.; OLIVEIRA, L. R. (2002) - Gestão de Ambiente Virtual de Ensino à Distância: Estudo de Caso Curso On-Line de Base de Dados na Gestão Empresarial. In. XXII ENEGEP, 2002, Curitiba, PR. Anais de Resumos: XXII Encontro Nacional de Engenharia de Produção. ABEPRO. Porto Alegre.

DRUCKER, P. (2000) - E-ducação. Revista Exame, São Paulo, Vol. 34, nº 12, ed. 716, p.64-67.

EISENBARTH, G. (2003) The online education market. On the Horizon, Vol. 11, No. 3, p. 9-15.

GARRISON, D. R. (1985) - Three generations of technological innovations in distance education. Distance Education, Vol. 6, n 2, pp. 235-241.

HIDDINK, G. W. (2001) - ADILE: Architeture of a Database-Supported Learning Environment. Journal of Interactive Learning Research. Vol. 12 (2/3), pp. 301-319.

KEEN, P. G. W. (1996) - Guia Gerencial para a tecnologia de informação: conceitos essenciais e terminologia para empresas e gerentes. Campus. Rio de Janeiro.

LÉVY, P. (1999) - Cibercultura. Esfera. São Paulo.

LEWIS, L; SNOW, K.; FARRIS, E.; LEVIN, D. (1999) - Distance Education at postsecondary education institutions: 1997-98. National Center for Education Statistics Report No. 2000-13. US Department of Education. Washington, DC.

MENA, M.; RODRÍGUEZ, L. E.; DIEZ, M. L. (2001) - La Educación a Distancia: Una Propuesta para el Diseño de Proyectos. IN: MISOCZKY, M. C. A.; CARVALHO, N. M.; OLIVO, V. (orgs.). Educação a Distância: Reflexões Críticas e Experiências em Saúde. Dacasa Editora. Porto Alegre.

MIODUSER, D.; NACHMIAS, R.; OREN, A.; LAHAV, O. (1999) - Web-based learning environments (WBLE): Current implementation and evolving trends. J. Network and Computer Appl. Vol. 22, pp. 233-247.

MOORE, M. G.; KEARSLEY, G. (1996) - Distance education: a systems view. Walsworth Publishing Company. Belmont (USA).

MÜlleR, C. J.; CORTIMIGLIA, M. N.; GABRIELli, L. V.; KAPPEL, A. M. (2003) - Gerenciamento de Processos e Indicadores em Educação à Distância. In. XXIII ENEGEP, 2003, Ouro Preto, MG. Anais de Resumos: XXIII Encontro Nacional de Engenharia de Produção. ABEPRO. Porto Alegre.

MUTULA, S. M. (2002) - E-learning initiative at the University of Botswana: challenges and opportunities. Campus-Wide Information Systems, Vol. 19, n 3, pp. 99-109.

NACHMIAS, R.; MIODUSER, D.; OREN, A.; LAHAV, O. (1999) - Taxonomy of educational Web-sites - A tool for supporting research, development and implementation of web-based learning. International Journal of Educational Telecommunications, Vol. 5, n 3, pp. 193-210.

NGANI, C. U.; FURNELL, S. M.; GHITA, B. V. (2002) - Assessing the global accessibility of the Internet. 
Internet Research: Eletronic Networking Applications and Policy, Vol. 12, $\mathrm{n}^{\circ}$ 4, pp. 329-338.

OLIVEIRA, L. R.; CORTIMIGLIA, M. N. (2003) - Administering a Web-Based Course on Database Technology. Online Journal Of Distance Learning Administration, http://www.westga.edu, Vol. 6, no 4.

REITZ, D. S. (2003) - Abordagem Ergonômica de Avaliação de Websites no Âmbito da Educação à Distância. Trabalho de Conclusão de Mestrado Profissionalizante em Engenharia - Escola de Engenharia, UFRGS.

SCHLEMMER, E.; FAGUNDES, L. C. (2001) - Uma Proposta para Avaliação de Ambientes Virtuais de Aprendizagem na Sociedade em Rede. Informática na Educação: Teoria \& Prática. Vol. 4, nº 2, pp. 25-36.

SHEREMETOV, L.; ARENAS, A. G. (2002) - EVA: an interactive Web-based collaborative learning environment. Computers \& Education, Vol. 39, pp. 161-182.

SIVAKUMAR, S. C.; ROBERTSON, W. (2004) - Developing an integrated Web engine for online Internetworking education: a case study. Internet Research, Vol. 14, No. 2, p. 175-192.

SLATOR, B. M.; JUELL, P.; McCLEAN, P. E.; SAINI-EIDUKAT, B.; SCHWERT, D. P.; WHITE, A. R.; HILL, C. (1999) - Virtual environments for education. J. Network and Computer Appl. Vol. 22, pp. 161-174.

TIGHT, M. (1998) - Lifelong Learning: Opportunity or Compulsion? British Journal of Educational Studies, Vol. 46, no 3, pp. 251-263.

TU, C. (2000) - Critical examination of factors affecting interaction on CMC. Journal of Network and Computer Applications. Vol. 23, pp. 39-58.

U.S. DEPARTMENT OF EDUCATION. (1999) - Post-secondary education quick info system: Survey on distance education at post-secondary education institutions. National Center for Education Statistics. Disponível on-line em http://nces.ed.gov, último acesso em 24/03/2004.

. (2002) - A Profile of Participation in Distance Education: 1999-2000. National Center for Education Statistics. Disponível on-line em http://nces.ed.gov, último acesso em 24/03/2004.

VODANOVICH, S. J.; PIOTROWSKI, C. (2001) - Internet-Based Instruction: A National Survey of Psychology Faculty. Journal of Instructional Psychology. Vol. 28, no 4, pp. 253-255.

WEB-BASED EDUCATION COMISSION. (2000) - The power of the Internet for learning: Report of the WebBased Education Commission to the President and Congress of the United States. The Web-Based Education Commission. Washington, D.C. Disponível on-line em http://www.ed.gov/offices/AC/WBEC/FinalReport/WBECReport.pdf, último acesso em 24/03/2004.

Artigo Recebido em 24/08/04 e aceito para publicação em 15/06/05. 